\title{
SOME INTERSECTION PROPERTIES OF CONVEX BODIES
}

\author{
G. D. CHAKERIAN AND S. K. STEIN
}

1. Introduction. By a convex body we shall mean a compact, convex subset of $n$-dimensional Euclidean space $E_{n}$, having interior points. If $A, B \subset E_{n}$, then $A$ and $B$ are homothetic if $B$ is a translate of $\lambda A$, for some $\lambda>0$.

Helly's Theorem asserts the following: given a family of convex bodies in $E_{n}$ such that each $n+1$ intersect, then they all intersect. If we assume only that each pair of sets of the family intersect, then a conclusion as strong as that of Helly's Theorem cannot be reached in general. If, however, a pairwise intersecting family of homothets of a fixed body is given, then the family can be partitioned into a finite number of subfamilies such that each subfamily intersects (see [1], [2]). Following [2], we associate with a convex body $K$ two numbers, $h(K)$ and $H(K)$, defined as follows: $h(K)$ is the least cardinal $r$ such that whenever $\mathcal{F}$ is any family of pairwise intersecting translates of $K$, then there exist $r$ points such that each member of $\mathcal{F}$ contains at least one of them. $H(K)$ is the least cardinal $r$ such that whenever $\mathfrak{F}$ is any family of pairwise intersecting translates of sets homothetic to $K$, then there exist $r$ points such that each member of $\mathcal{F}$ contains at least one of them.

Obviously $h(K) \leqq H(K)$. Equality is possible - for example, if $P$ is a parallelopiped, then $h(P)=H(P)=1$ (see [2, p. 156]). In $\S 3$ we shall prove that if $T$ is a triangle, then $h(T)=H(T)=3$. Strict inequality is also possible - for example, if $C$ is a circular disk, then $h(C)$ $=3<4=H(C)$ (see [1, p. 144]). It is proved in [2] that if $K \subset E_{2}$ is centrally symmetric, then $h(K) \leqq 3$ and $H(K) \leqq 7$, and conjectured that $h(K) \leqq 3$ for any convex body $K \subset E_{2}$. Let $h(n)=\sup h(K)$, $K \subset E_{n}$, and $H(n)=\sup H(K)$. It is proved in [2] that $H(n)$, and hence $h(n)$, is finite, and upper bounds are given. We shall prove in $\$ 2$ that

$$
h(n) \leqq n^{n}, \quad n \geqq 1 .
$$

Thus $h(2)$ is either 3 or 4 . (The results of C. A. Rogers-see [1, p. 147 - give upper bounds for $h(n)$ which are much better than $n^{n}$ for large $n$.)

The proof of (1) depends on the following lemma, proved in $\$ 2$, which is of interest in its own right.

Received by the editors June 22, 1966. 
Lemma 1. Let $K$ be a convex body in $E_{n}$. Then $K$ contains a parallelopiped $P$ such that some translate of $n P$ contains $K$.

Let $Q_{n}$ be the collection of equivalence classes of compact convex subsets of $E_{n}$, where two sets are identified if and only if one is a regular affine image of the other. A metric can be defined for $Q_{n}$ as follows. If $K_{1}$ and $K_{2}$ are convex bodies, let $\Lambda=\inf \left\{\lambda \geqq 0: T\left(K_{1}\right) \subset K_{2}\right.$ and some translate of $\lambda T\left(K_{1}\right)$ contains $K_{2}$, where $T$ ranges over regular affine maps $\}$. Let $\mu\left(K_{1}, K_{2}\right)=\log \Lambda$. If $K_{1}$ and $K_{2}$ are representatives of the equivalence classes $\left[K_{1}\right]$ and $\left[K_{2}\right]$ respectively of $a_{n}$, then we define $\mu\left(\left[K_{1}\right],\left[K_{2}\right]\right)=\mu\left(K_{1}, K_{2}\right)$. It is easily verified that $\mu$ is a metric on $Q_{n}$ (see [3, p. 236]). Lemma 1 implies that if $C$ is a cube and $K$ any convex body in $E_{n}$, then $\mu(C, K) \leqq \log n$. Moreover, if $T$ is a simplex, $\mu(C, T)=\log n$. Thus $\max \mu(C, K)=\log n$. This settles a question raised in $[3$, p. 259].

We conclude in $\$ 4$ with a special result about $h(n)$.

2. Proof of Lemma 1. Let $P$ be a parallelopiped of minimum volume containing $K$ (such a parallelopiped exists by standard compactness arguments and is nondegenerate since $K$ has interior points). We shall show that a translate of $(1 / n) P$ is contained in $K . P$ consists of points $\mathrm{x}$ of the form $\mathrm{x}=\mathrm{x}_{0}+\sum_{i=1}^{n} \alpha_{i} \boldsymbol{a}_{i}$, where $0 \leqq \alpha_{i} \leqq 1$, $i=1, \cdots, n$, and $a_{1}, \cdots, a_{n}$ are $n$ independent vectors $(n$ independent "edges" of $P$ ). Facets $F_{1}, \cdots, F_{n}$ of $P$ are defined as follows: $F_{j}=\left\{\mathrm{x}_{0}+\sum_{i=1}^{n} \alpha_{i} a_{i} \in P: \alpha_{j}=0\right\}$. Corresponding to each facet $F_{j}$ we have an opposite facet $F_{j}^{\prime}=a_{j}+F_{j}=\left\{x_{0}+\sum_{i=1}^{n} \alpha_{i} a_{i} \in P: \alpha_{j}=1\right\}$. Now we claim that for each $j=1, \cdots, n$ there exists $\boldsymbol{p}_{j} \in K$ such that also $\boldsymbol{p}_{j}+\boldsymbol{a}_{j} \in K$. This is seen as follows. Let $K^{*}=\boldsymbol{a}_{j}+K$. If $K^{*} \cap K=\varnothing$, then there is a hyperplane $H$ separating $K$ and $K^{*}$. If we keep the hyperplanes carrying those facets $F_{i}, F_{i}^{\prime}$ with $i \neq j$, and replace the hyperplane carrying $F_{j}^{\prime}$ by $H$, and the hyperplane carrying $F_{j}$ by $-a_{j}+H$, then this new set of hyperplanes encloses a parallelopiped $S$ containing $K$ and having the same volume as $P$. But $S$ has a pair of opposite facets free of points of $K$; hence $S$ contains a parallelopiped of smaller volume which in turn contains $K$, contradicting the minimum property of $P$. Thus $K^{*} \cap K \neq \varnothing$, and there exists a point $\boldsymbol{p}_{j} \in K$ such that $\boldsymbol{a}_{j}+\boldsymbol{p}_{j} \in K$, as claimed. Now to each of the $2^{n} n$-tuples $\Delta=\left(\delta_{1}, \cdots, \delta_{n}\right)$ where each $\delta_{i}$ is either 0 or 1 , there corresponds the unique vertex of $P$ of the form $x_{0}+\sum_{i=1}^{n} \delta_{i} a_{i}$. For each such $\Delta$, let $\boldsymbol{q}_{\Delta}=(1 / n) \sum_{i=1}^{n}\left(\boldsymbol{p}_{i}+\delta_{i} \boldsymbol{a}_{i}\right)$. Note that each $\boldsymbol{q}_{\Delta}$ is a point of $K$, being the centroid of points of $K$. Writing $q_{\Delta}$ $=(1 / n) \sum_{i=1}^{n} p_{i}+(1 / n) \sum_{i=1}^{n} \delta_{i} a_{i}$, one sees that the $q_{\Delta}$ form the 
vertices of a translate of $(1 / n) P$. Thus the convex hull of the set of $\boldsymbol{q}_{\Delta}$ is a translate of $(1 / n) P$ contained in $K$. This completes the proof.

Proof of (1). One has $h(1)=1$. (This is just Helly's Theorem in $E_{1}$.) Let $n \geqq 2$, and let $K$ be a convex body in $E_{n}$. Let $P^{\prime} \subset K \subset P$, where $P^{\prime}$ is a parallelopiped and $P$ is a translate of $n P^{\prime}$. Let $\mathcal{F}=\{\boldsymbol{q}+K: \boldsymbol{q} \in Q\}$ be a pairwise intersecting family of translates of $K$. Then $\mathfrak{F}^{*}=\{\boldsymbol{q}+P: \boldsymbol{q} \in Q\}$ is a pairwise intersecting family of translates of $P$; hence there exists a point common to all the $q+P$, $q \in Q$ since $h(P)=1$ (see $[2$, p. 156]). It follows that $Q$ is contained in a translate of $P$. But $P$ can be covered by $n^{n}$ translates of $P^{\prime}$ in the obvious way, so that $Q$ can be covered by $n^{n}$ translates of $-K$. Thus there exist $l=n^{n}$ points $p_{1}, \cdots, p_{l}$, such that each $q \in Q$ is contained in one of the $\boldsymbol{p}_{i}-K$. That is, for each $\boldsymbol{q} \in Q, \boldsymbol{p}_{i} \in \boldsymbol{q}+K$, for some $i$. Thus each member of $\mathcal{F}$ contains at least one of the $\boldsymbol{p}_{\mathbf{1}}, \cdots, \boldsymbol{p}_{l}$; so $h(K) \leqq n^{n}$. Hence $h(n) \leqq n^{n}$, as was to be proved.

3. If $T$ is a triangle, $H(T)=h(T)=3$.

Proof. Considering $\mathcal{F}=\{\boldsymbol{t}+T: \boldsymbol{t} \in T\}$ one sees that $h(T) \geqq 3$. Thus, since $h(T) \leqq H(T)$, it suffices to show that $H(T) \leqq 3$. Let $A, B$, and $C$ denote the vertices of $T$, and let $F$ be a pairwise intersecting family of translates of triangles homothetic to $T$. If each member of $\mathcal{F}$ is projected orthogonally on to a line $l$ perpendicular to side $A B$, a pairwise intersecting family of segments on $l$ is obtained. There is a point $P$ common to all these segments. The line $l_{1}$ through $P$ perpendicular to $l$-and hence parallel to $A B$-intersects all the members of $\mathcal{F}$. Similarly there are lines $l_{2}$ and $l_{3}$ parallel to $B C$ and $C A$ respectively and intersecting all the members of $\mathcal{F}$. If $l_{1}, l_{2}, l_{3}$ intersect in a point, then any triangle intersecting all three lines and homothetic to $T$ must contain that point; hence all members of $\mathcal{F}$ would have a point in common. If $l_{1} \cap l_{2} \cap l_{3}=\varnothing$ and if $l_{1} \cap l_{2}, l_{1} \cap l_{3}, l_{2} \cap l_{3}$ are the vertices of a triangle $T^{\prime}$ homothetic to $T$, then any triangle homothetic to $T$ and intersecting all three lines must contain $T^{\prime}$, and again all members of $\mathfrak{F}$ would have a point in common. The only other possibility is that the points $l_{1} \cap l_{2}, l_{1} \cap l_{3}, l_{2} \cap l_{3}$, are the vertices of a triangle $T^{\prime \prime}$ homothetic to $-T$. It is then easy to verify that any triangle homothetic to $T$ and intersecting all three lines must contain at least one of the midpoints of the sides of $T^{\prime \prime}$. (The reader may draw a picture.) Hence we have three points such that each member of $\mathcal{F}$ contains at least one of those points. This completes the proof.

4. The following consequence of Helly's Theorem is well known and easy to prove (see $[1$, p. 110]). Let $\mathfrak{F}$ be a family of convex bodies 
in $E_{n}$, and let $L$ be a convex body. Suppose that, given any $n+1$ members of $\mathcal{F}$, there exists a translate of $L$ intersecting all of them. Then there exists a translate of $L$ intersecting all members of $F$.

In the above, we may replace the word "intersecting" by "containing" or by "contained in." The following analogous result is of interest.

Let $\mathcal{F}$ be a family of translates of a convex body $K$ in $E_{n}$, and let $L$ be a convex body. Suppose that $\mathcal{F}$ has the property that, given any pair of its members, some translate of $L$ intersects both. Then there exist $l=h(n)$ translates, $L_{1}, \cdots, L_{l}$, of $L$ such that given any member of $\mathcal{F}$, one of the $L_{1}, \cdots, L_{l}$ intersects that member. In the above, we may replace the word "intersects" by "contains" or by "is contained in."

Proof. For each $K_{\alpha} \in \mathcal{F}$, let $K_{\alpha}^{*}=\left\{x: x+L\right.$ intersects $\left.K_{\alpha}\right\}$. Then the $K_{\alpha}^{*}$ form a family $\mathcal{F}^{*}$ of translates of a fixed convex set. $\mathfrak{F}^{*}$ is pairwise in tersecting, since, if $K_{\alpha}^{*}, K_{\beta}^{*} \in \mathcal{F}^{*}$ and if $\mathrm{x}+L$ intersects both $K_{\alpha}$ and $K_{\beta}$, then $x \in K_{\alpha}^{*} \cap K_{\beta}^{*}$. Hence there exist $l=h(n)$ points $p_{1}, \cdots, p_{l}$ such that each member of $\mathfrak{F}^{*}$ contains one of them. But when $K_{\alpha}^{*}$ contains $\boldsymbol{p}_{i}$, that means $\boldsymbol{p}_{i}+L$ intersects $K_{\alpha}$. This proves the theorem with "intersects" in the statement. To complete the proof, replace the word "intersects" by "contains" or by "is contained in" everywhere except in the last sentence of the proof.

\section{REFERENCES}

1. L. Danzer, B. Grünbaum and V. Klee, Helly's Theorem and its relatives, Proc. Sympos. Pure Math., Vol. 7, pp. 101-180, Amer. Math. Soc., Providence, R. I., 1963.

2. B. Grünbaum, On intersections of similar sets, Portugal. Math. 18 (1959), 155164.

3. - Measures of symmetry for convex sets, Proc. Sympos. Pure Math., Vol. 7, pp. 233-270, Amer. Math. Soc., Providence, R. I., 1963.

University of California, Davis 